\title{
Tactile Information Presentation In The Cockpit
}

\author{
Henricus A. H. C. van Veen \& Jan B.F. van Erp \\ TNO Human Factors \\ P.O. Box 23 \\ 3769 ZG Soesterberg \\ The Netherlands \\ +31346356449 \\ vanVeen@tm.tno.nl
}

\begin{abstract}
This paper describes two aspects of the application of tactile information presentation in the cockpit. The first half of the paper discusses why the tactile channel might be used instead of, or in addition to, the more common visual and auditory channels. It lists several categories of information used in cockpits and explores their appropriateness for tactile stimulation. The second half of the paper briefly describes an experiment on the perception of vibro-tactile stimuli under high G-load conditions (in a centrifuge). It is concluded that the perception of vibrotactile stimulation on the torso is not substantially impaired during high G-load conditions, at least up to $6 \mathrm{G}$.
\end{abstract}

\section{Keywords}

Tactile, tactile perception, tactile channel, vibro-tactile, cockpit, G-load.

\section{WHY WE SHOULD USE TACTILE INFORMATION PRESENTATION IN COCKPITS}

The tactile channel is a relatively neglected information channel in display research, also in cockpit displays. Worldwide only a few groups have current research programmes in this area (e.g., see [2,3,4]). Visual displays dominate the design of cockpits, and auditory displays are increasingly being used as well. Tactile displays, however, are virtually absent in cockpits. Nevertheless, there are many situations in which the tactile channel can become an important or even vital alternative, because the visual (and/or auditory) channel is not available, not adequate, or overloaded (e.g., see [2]). Some relevant considerations (some more speculative than others) are:

- The enormous amount of information that is available to the pilot is offered primarily in a visual format. The limits of the visual processing capabilities of pilots constitute a real design constraint in the development of new cockpits.
- The view on the outside world in a cockpit (field-ofregard) is generally and obviously limited, because only a part of the cockpit is transparent. Systems that use forms of indirect sight (such as camera-monitor systems) can be used to overcome this limitation, but always have a restricted instantaneous field-of-regard.

- High G-loads, such as experienced in fighter jets, can severely degrade visual perception. Maybe tactile perception does not suffer from this problem.

- Visual information can be hard to interpret, e.g., when representing spatial information (3D) on 2D visual displays. Presenting such information to the skin might reduce those interpretation problems. The surface of the skin is a 2D surface like a visual display, but unlike such a display it is also a closed manifold embedded in a 3D space (sphere topology), and can therefore be used to represent part of the 3D spatial relations directly, namely, directions in 3D space.

- Pilots commonly experience visual and visualvestibular illusions, some of them resulting in disorientation. It is conceivable that tactile stimulation could support the pilot in recognising the occurrence of such illusions and in avoiding their negative effects on performance.

- Visual attention is usually restricted to a single entity (with the exception of moving objects). Thus, tracking multiple visual information sources in parallel is probably limited. How this translates to the tactile modality (and multi-modality) is not exactly known, but there are some indications that tactile attention may be directed to more than one location concurrently.

The above considerations are really examples of the earlier mentioned arguments for using tactile instead of, or in addition to, visual/auditory stimulation: non-availability, inadequacy, and processing overload. Another dimension 
along which this problem needs to be studied is the type of information that is suitable for presentation via the tactile channel. At least four relevant categories of information present in cockpits can be identified:

1. Geometric information: the projection of spatially organised information on a spatially organised medium. Major examples are:

- Directions in 3D space. Waypoints, other aircraft, targets, etc., can all be characterised by a direction in 3D space. These directions change rapidly when the pilot moves through the environment. Information of this type could be presented continuously, or when the pilot asks for it, or could be used as a cueing/warning system (e.g., see [1]).

- Reference frames. An artificial horizon can be represented in the tactile modality by stimulating those parts of the torso that form the intersection of the torso with the actual horizon.

- Borders in the sky. Borders in the sky can originate from airspace rules (restricted areas, minimum height, etc.), from course restrictions (tunnel-in-the-sky) or course planning, from missions (e.g., dropzones), and probably from many other causes. When such borders are interpreted as surfaces in 3D space, pilots can be made aware of them by appropriate tactile stimulation during approach (e.g., tactile stimulation of the relevant side of the body with increasing intensity or frequency upon approach) and passing (e.g., similar to the type of stimulation suggested for indicating reference frames) of such surfaces.

2. Warning signals: the principles for warning hierarchies in use for controlling visual and auditory warnings can also be developed for the tactile domain. However, a multi-modal approach - which modality should be used with which strength and form - would probably be even more powerful.

3. Coded information: all other types of information not included in the first two categories can of course be projected to the tactile domain in a coded form. Examples are flight manoeuvre related data, such as altitude, speed, attitude, and feedback signals in hovering manoeuvres, but also information like fuel supply, identification of radar signals, time-to-contact, payload information, etc. Optimal ways of coding need to be developed.
4. Communication: The tactile modality might also be used for simple but effective forms of communication between crewmembers or between members of a formation. Such a communication channel might be useful for covert operations, for communicating simultaneity between individuals, for indicating directions of danger by remote tactile stimulation on another persons body, etc.

These contemplations can be used to derive a human factors research agenda for investigating the advantages and disadvantages of using tactile versus visual and auditory information presentation in the cockpit. For instance, additional studies on multi-modal attention and processing capacity need be performed before it can be confirmed that tactile stimulation can be used to overcome current processing overload problems. The second half of this paper discusses an experiment along one of the other lines, namely vibro-tactile perception under high G-load.

\section{VIBRO-TACTILE PERCEPTION UNDER G-LOAD}

Earlier work at TNO Human Factors has shown that tactile displays can be used effectively for presenting spatial information, such as the direction of waypoints. For these studies, a tactile display was designed that allows for an intuitive way of presenting external directions by means of vibro-tactile stimulation. This display is worn on the torso and can be extended to a maximum of 128 actuators distributed over the body.

The current pilotstudy aims to probe the perception of vibro-tactile stimulation under high G-load. The motivation is that the application of tactile displays in fighter jets would be much more valuable when pilots can continue to perceive and process tactile stimulation under high G-load conditions where the visual channel degrades strongly or becomes completely unusable. The main factors that potentially hinder the tactile perception during high G-load are: mechanical aspects of human skin receptors, pressure suits and straining procedures, reduced attention for tactile stimulation caused by the high stress and workload levels, and mechanical aspects of the actuators used for the stimulation (our display was not designed for high-G applications). 


\section{Methods}

The experiment was conducted in the centrifuge of the Aeromedical Institute (AMI) in Soesterberg, The Netherlands, under supervision of a medical doctor. Each of the four participants was subjected to a number of $\mathrm{G}$ profiles (see table 1), starting with a so-called relaxed $\mathrm{G}$ tolerance test. During all profiles, subjects were wearing a simple version of the tactile display, consisting of three or four tactile actuators mounted on the left and right side of the torso. The actuators were activated as a group (left or right) in a $100 \mathrm{~ms}$ on $-200 \mathrm{~ms}$ off rhythm, during 6 seconds maximum. Subjects had to press one of two buttons (left or
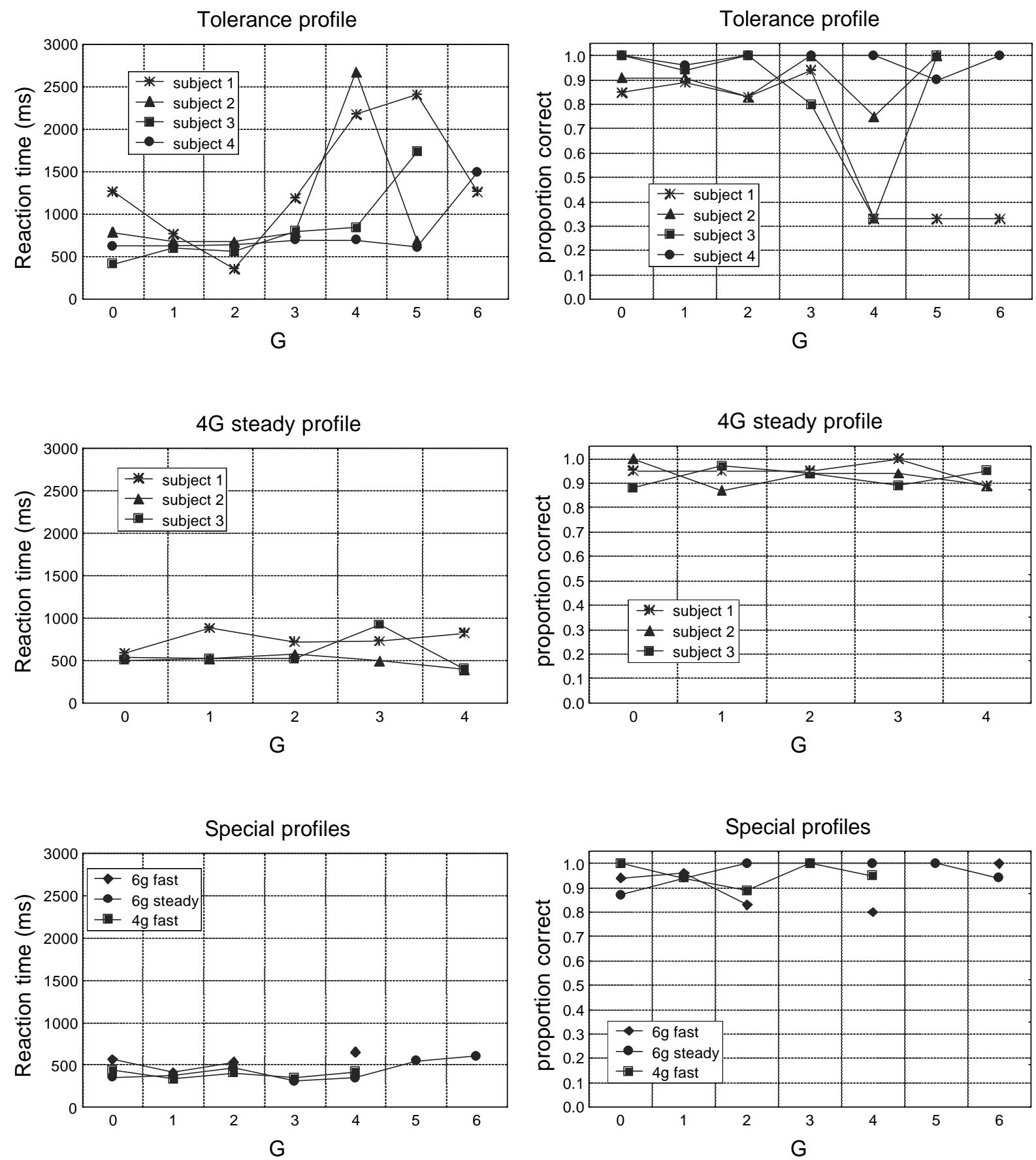

Figure 1: Reaction time (left column) and proportion correct responses (right column) as a function of the G-load for different G-profiles. 
right), immediately upon detection of tactile stimulation at either the left or right side of their torso. Because of the obviously short durations of the G-profiles, the next trial started almost immediately after the subject responded. All four subjects were male, between 30-40 years old, member of either two institutes, and participated voluntarily. When considered necessary, a medical examination was conducted before the experiment. During the experiment, a medical doctor continuously monitored the subjects by means of verbal communication, video monitoring, and ECG (electrocardiogram) monitoring. Subjects either had previous experience with G-loads $(1,4)$ or vibro-tactile stimulations $(2,3)$.

\begin{tabular}{|l|l|l|}
\hline Profile & Description & Subjects \\
\hline Tolerance & $\begin{array}{l}\text { 0.33 G/sec increase of G-load, } \\
\text { aborted when subject experiences } \\
\text { troubles with vision. }\end{array}$ & All \\
\hline 4G steady & $\begin{array}{l}0.33 \mathrm{G} / \mathrm{sec} \text { increase of G-load up to } \\
\text { 4G, then steady for 30sec. Subject } \\
\text { strains leg muscles. }\end{array}$ & $\begin{array}{l}1, \\
2 \text { (twice), } \\
3\end{array}$ \\
\hline 4G fast & Similar to 4G steady, but with 1G/sec. & 4 \\
\hline 6G fast & $\begin{array}{l}1 \text { G/sec increase of G-load up to 6G. } \\
\text { Subject wears pressure suit (legs } \\
\text { only) and performs straining. }\end{array}$ & 4 \\
\hline 6G steady & $\begin{array}{l}\text { Similar to 6G fast, but with additional } \\
15 \text { sec steady at 6G. }\end{array}$ & 4 \\
\hline
\end{tabular}

Table 1: Five different G-profiles used during the experiment.

\section{Results \& Conclusions}

Figure 1 summarises the results. The results obtained with the G-tolerance profiles show stable response levels (reaction time and percentage correct) up till about 3G, but decreased performance close to the individual G-tolerance levels. This initial performance reduction at higher Glevels disappeared completely in subsequent G-profiles, possibly due to the familiarisation with the task and physiological condition. Note that this is even true for those G-levels that are close to the individual relaxed $\mathrm{G}$ tolerance levels. Reaction times stabilise at around 500 msec (subject dependent) and percentages correct response are invariably high (between 85 and 100\%).

Apparently, the perception of vibro-tactile stimulation on the torso is not substantially impaired during high G-load conditions, at least up to 6G. Note that the torso is certainly not the most sensitive part of the body with respect to vibro-tactile stimulation, in terms of detection thresholds and spatial resolution for instance. Secondly, it seems that there are no differences between the conditions with and without a pressure suit and extended straining (subject 4 in the $4 \mathrm{G}$ fast versus $6 \mathrm{G}$ fast and $6 \mathrm{G}$ steady conditions).

\section{ACKNOWLEDGEMENTS}

TNO Human Factors kindly acknowledges the support from our colleagues at the Aeromedical Institute in Soesterberg, The Netherlands, in conducting the G-load experiments in their centrifuge.

\section{REFERENCES}

1. Erp, J. B. F. van (2000, in press). Direction determination with vibro-tactile stimuli presented to the torso: a search for the tactile ego-centre. Report TM-00-B. Soesterberg, The Netherlands: TNO Human Factors.

2. Rupert, A. H. (1999). Haptic solution to directed energy threats. NATO RTO HFM Symposium on "Countering the directed energy threat: Are closed cockpits the ultimate answer?", Antalya, Turley, 26-28 April 1999. RTO-MP-30, AC/323(HFM)TP/10.

3. Raj, A. K., McGrath, B. J., Rochlis, J., Newman, D. J. \& Rupert, A. H. (1998). The application of tactile cues to enhance situation displays. 3rd Annual Symposium \& Exhibition on Situational Awareness in the Tactical Air Environment, Patuxent River, MD, 77-84.

4. Raj, A. K., Suri, N., Braithwaite, M. G . \& Rupert, A. (1998). The tactile situation awareness system in rotary wing aircraft: Flight test results. NATO RTO HFM Symposium on "Current aeromedical issues in rotary wing operations”, San Diego, USA, 19-21 October 1998. RTO-MP-19. 